\title{
CpG island methylation of BNIP3 predicts resistance against S-1/CPT-11 combined therapy in colorectal cancer patients
}

\author{
MASATSUGU HIRAKI, YOSHIHIKO KITAJIMA, YUJI NAKAFUSA, JUN NAKAMURA, \\ KAZUYOSHI HASHIGUCHI, KENJI SUMI, HIROKAZU NOSHIRO and KOHJI MIYAZAKI \\ Department of Surgery, Saga University Faculty of Medicine, 5-1-1 Nabeshima, Saga 849-0937, Japan
}

Received July 30, 2009; Accepted September 4, 2009

DOI: 10.3892/or_00000622

\begin{abstract}
Aberrant gene methylation is frequently observed in various cancers and plays an important role in carcinogenesis, cancer progression and drug responsiveness. The aim of this study is to identify colorectal cancer specific gene methylation determining chemosensitivity to S-1/CPT-11 therapy. The gene methylation of CHFR, p16, RUNX3, Ecadherin, MGMT, hMLH1, ABCG2, UGT1A1 and BNIP3 genes were analyzed in 27 colorectal cancer tissues by quantitative methylation-specific PCR (q-MSP). All 27 patients were postoperatively treated by S-1/CPT-11 therapy targeting the metastatic lesion and the recurrent tumor. Thereafter, the patients were divided into a responder group (RG) or a non-responder group (NRG) according to the effect of the chemotherapy. There were 13 cases of RG $(48.1 \%)$ and 14 cases of NRG (51.9\%). The methylation level in CHFR, RUNX3 and BNIP3 was significantly higher in cancer lesions in comparison to the non-cancerous lesion. Only methylation of the BNIP3 gene was significantly higher in primary cancer tissue of the NRG than the RG. The correlation between the BNIP3 methylation status and time to progression (TTP) suggested that the low methylation group $(n=16)$ resulted in a significantly longer TTP, in comparison to the high methylation group $(\mathrm{n}=11 ; \mathrm{P}=0.004)$. The methylation level of BNIP3 showed a significant inverse correlation with the mRNA expression suggesting the DNA methylation suppressed BNIP3 expression $(r=-0.466, P=0.021)$. In conclusion, BNIP3 gene methylation is a possible marker predicting a poor response to the $\mathrm{S}-1 / \mathrm{CPT}-11$ combined therapy in colorectal cancer.
\end{abstract}

\section{Introduction}

Colorectal cancer (CRC) is one of the most common malignancies and the third leading cause of cancer related

Correspondence to: Dr Kohji Miyazaki, Department of Surgery, Saga University Faculty of Medicine, 5-1-1 Nabeshima, Saga 8498501, Japan

E-mail: miyazak2@cc.saga-u.ac.jp

Key words: colorectal cancer, methylation, BNIP3, chemoresistance, S-1, CPT-11, irinotecan deaths (1). Surgical resection is the most powerful treatment for the complete cure of CRC patients. However, a surgical resection is occasionally impossible due to the advanced stage in several patients. Furthermore, a local or distant tumor recurrence may develop even if a curative resection of the primary tumor is performed. Therefore, effective chemotherapy is an important strategy for prolonging the survival of patients with CRC. Various drugs have been developed and administered to patients with advanced or recurrent CRC. However, an ineffective drug allows further growth of the tumor and results in an unfavorable outcome. If the drug efficacy is predicted before the treatment, the information will help to select the optimal anticancer drugs individually, and socalled tailor-made-therapy might improve the outcome of chemotherapy.

Several predictive factors for chemosensitivity have been investigated for several solid tumors. Thymidylate synthase (TS), dihydropyrimidine dehydrogenase (DPD) and ortate phosphoribosyltransferase (OPRT), which are critical enzymes in the metabolism of 5-fluorouracil (5-FU) could be important factors determining chemosensitivity to 5-FU or the modified agent, S-1 (2-4). Besides, aberrant promoter methylation of tumor suppressor genes, DNA repair genes, and cell cycle checkpoint genes are frequently found in human primary CRC and show a significant correlation with carcinogenesis, tumor progression and chemosensitivity (5-9). There is a relationship between aberrant gene methylation and chemosensitivity to anticancer drugs, for example CHFR and microtubule inhibitors in gastric cancer tissues and cell lines, p16 and 5-FU-based therapy in gastric cancer tissues, hMLH1 and 5-FU-based therapy in colorectal cancer tissues (10-12).

We recently reported that a phase I/II study of Tegafur, gimeracil, oteracil potassium (S-1) plus CPT-11 therapy in 33 metastatic CRC patients demonstrated a response rate of $58 \%$ (13). The present study examined DNA methylation of 9 cancer related genes using primary CRC tissues from 27 patients. The nine genes include CHFR (checkpoint with forkhead and ring finger domains), p16 (cyclin-dependent kinase inhibitor 2A), RUNX3 (runt-related transcription factor 3), E-cadherin, MGMT (O'6-methylguanin DNA methyltransferase), hMLH1 (mutL homolog 1), ABCG2 (ATPbinding cassette, sub-family G, member 2), UGT1A1 (UDP glucuronosyltransferase 1 family, polypeptide A1) and BNIP3 (BCL2/adenovirus E1B $19 \mathrm{kDa}$ interacting protein 3), which all show frequent methylation in several cancer types (8,10-12,14-17). Three genes expressing the CRC specific 
Table I. Primer and probe sequence of LightCycler system for q-MSP.

\begin{tabular}{|c|c|c|}
\hline Gene & Primer sequence & Refs. \\
\hline CHFR & $\begin{array}{l}\text { Forward: TTTCGTGATTCGTAGGCGAC } \\
\text { Reverse: CGACAACTAAAACGAAACCGA } \\
\text { Probe: 5'-FAM-CGCGAAAATAAACGCGTAAAAAACGCTCG-3'BHQ }\end{array}$ & ( 11, this study) \\
\hline p16 & $\begin{array}{l}\text { Forward: TGGAATTTTCGGTTGATTGGTT } \\
\text { Reverse: AACAACGTCCGCACCTCCT } \\
\text { Probe: 5'-FAM- ACCCGACCCCGAACCGCG-3'BHQ }\end{array}$ & $(20)$ \\
\hline RUNX3 & $\begin{array}{l}\text { Forward: CGTTCGATGGTGGACGTGT } \\
\text { Reverse: GACGAACAACGTCTTATTACAACGC } \\
\text { Probe: 5'-FAM-CGCACGAACTCGCCTACGTAATCCG-3'BHQ }\end{array}$ & (21) \\
\hline E-cadherin & $\begin{array}{l}\text { Forward: AATTTTAGGTTAGAGGGTTATCGCGT } \\
\text { Reverse: TAACTAAAAATTCACCTACCGAC } \\
\text { Probe: 5'-FAM-CGCCCACCCGACCTCGCAT-3'BHQ }\end{array}$ & $(20,22)$ \\
\hline MGMT & $\begin{array}{l}\text { Forward: TTTCGACGTTCGTAGGTTTTCGC } \\
\text { Reverse: GCACTCTTCCGAAAACGAAACG } \\
\text { Probe: 5'-FAM-ACCAAATCGCAAACGATACGC-3'BHQ }\end{array}$ & ( 23, this study) \\
\hline hMLH1 & $\begin{array}{l}\text { Forward: CGTTATATATCGTTCGTAGTATTCGTGTTT } \\
\text { Reverse: CTATCGCCGCCTCATCGT } \\
\text { Probe: 5'-FAM-CGCGACGTCAAACGCCACTACG-3'BHQ }\end{array}$ & (20) \\
\hline ABCG2 & $\begin{array}{l}\text { Forward: TTGGGTAATTTGTGCGTTA } \\
\text { Reverse: CTACGAAAATCACCAAACGCTC } \\
\text { Probe: 5'-FAM-TTAATCGCCGTCACTAACCGA-3'BHQ }\end{array}$ & (this study) \\
\hline UGT1A1 & $\begin{array}{l}\text { Forward: TAGTTAAATATTAATTTGGTGTATCGA } \\
\text { Reverse: CCTAAAACTCCACAACCATAACG } \\
\text { Probe: 5'-FAM-CCTACCAAAAATTCGCCCTCTCC-3'BHQ }\end{array}$ & (this study) \\
\hline BNIP3 & $\begin{array}{l}\text { Forward: TAGGATTCGTTTCGCGTACG } \\
\text { Reverse: ACCGCGTCGCCCATTAACCGCG } \\
\text { Probe: 5'-FAM-CGTAAAATACGTATAACACGTACGAC-3'BHQ }\end{array}$ & ( 24, this study) \\
\hline B-actin & $\begin{array}{l}\text { Forward: TGGTGATGGAGGAGGTTTAGTAAGT } \\
\text { Reverse: AACCAATAAAACCTACTCCTCCCTTAA } \\
\text { Probe: 5'-FAM-ACCACCACCCAACACACAATAACAAACACA-3'BHQ }\end{array}$ & (20) \\
\hline
\end{tabular}

methylation were isolated through a quantitative methylationspecific PCR (q-MSP) method using 27 paired cancer tissues and the non-cancerous mucosa specimens. Subsequently, the gene methylation was examined in order to determine whether or not the methylation status correlates with the responsiveness to S-1/ CPT-11 therapy. The aim of this study was to identify CRC specific gene methylation determining the chemosensitivity to S-1/ CPT-11 therapy.

\section{Materials and methods}

Patients and tissue samples. Seventy-one CRC patients were treated by S-1/CPT-11 therapy from 2004 to 2008 at the Department of Surgery, Saga University Hospital. Twentyseven of the 71 patients underwent a surgical resection of the primary tumor. A metastatic lesion was concomitantly found during the surgery in 15 cases $(55.6 \%)$, whereas a recurrent tumor developed postoperatively in 12 cases $(44.4 \%)$. Informed consent was obtained from all the patients before the collection of the samples and the study protocol was approved by the Ethics Committee of Saga University, Faculty of Medicine. CRC tissues and non-cancerous tissues were obtained at surgery and stored at $-80^{\circ} \mathrm{C}$ until extraction of genomic DNA and RNA. The 27 patients included 10 (37.0\%) females and $17(63.0 \%)$ males. The mean age of the patients was 65.5 (ranging from 40 to 84 years). The maximal response to $\mathrm{S}-1 / \mathrm{CPT}-11$ therapy was assessed on the metastatic lesion or the recurrent tumor according to by the response evaluation criteria in solid tumors (RECIST) (18). Assessment of the response was based on the RECIST criteria, in which the 
Table II. Primer and probe sequence of LightCycler system for q-RT-PCR.

\begin{tabular}{lll}
\hline Gene & \multicolumn{1}{c}{ Primer sequence } & Refs. \\
\hline BNIP3 & Forward: CGCAGACACCACAAGATACC & (this study) \\
& Reverse: GAATATTTTCCGGCCGACTT \\
& Probe: 5'-FAM-GGGTATCTGTTTCAGAAGCCC-3'BHQ & (this study) \\
GAPDH & Forward: CGAGATCCCTCCAAAATCAA \\
& Reverse: TTCACACCCATGACGAACAT & \\
& Probe: 5'-FAM-CGCTGAGTACGTCGTGGAGTCCACT-3'BHQ \\
\hline
\end{tabular}

responder group (RG) included complete response (CR) and partial response (PR), while the non-responder group (NRG) indicated stable disease (SD) and progression disease (PD). The duration of S-1/CPT-11 effect was evaluated by the time to tumor progression (TTP; median: 11.0 months; range: 1.653.0 months). Fifteen of $27(55.6 \%)$ patients were treated by $\mathrm{S}-1 / \mathrm{CPT}-11$ therapy as the first treatment. The other 12 $(44.4 \%)$ patients had been treated by other regimens and underwent S-1/CPT-11 therapy as 2nd-4th line therapy. The pretreatment regimens included tegafur-uracil/leucovorin (LV) therapy (7 cases), 1-LV/5-FU therapy (5 cases), S-1 monotherapy (2 cases), tegafur-uracil monotherapy (1 case), and doxifluridine (5'-DFUR)/CPT-11 therapy (1 case). The treatment regimens and details were previously reported (13).

DNA extraction and sodium bisulfite modification and quantitative methylation-specific PCR. The genomic DNA from the tissue samples was isolated using a DNA extraction kit (EZ1 DNA tissue kit, Qiagen, Hilden, Germany). Bisulfite modification was carried out using $2 \mu \mathrm{g}$ of genomic DNA by MethylEasy $^{\mathrm{TM}}$ Xceed (Human Genetic Signitures, North Ryde, Australia) according to the manufacturer's instructions. Modified DNA was suspended in $100 \mu 1$ TE buffer. Twenty-seven primary CRC tissue specimens were analyzed for the methylation status of 10 genes (CHFR, p16, RUNX3, E-cadherin, MGMT, hMLH, ABCG2, UGT1A1, BNIP3 and $\beta$-actin as an internal marker). For q-MSP, an aliquot of $2 \mu \mathrm{l}$ was amplified by PCR using a primer set along with TaqMan probe specific to methylated sequences. A q-MSP (MethyLight) was carried out through LightCycler $^{\mathrm{TM}}$ instrument system (Roche, Mannheim, Germany) using the LightCycler ${ }^{\circledR}$ TaqMan ${ }^{\circledR}$ Master (Roche) as previously described (19). The primer sequences are shown in Table I $(11,20-24)$. After a denaturing step at $95^{\circ} \mathrm{C}$ for $10 \mathrm{~min}, \mathrm{PCR}$ amplification was performed with 45 cycles of $15 \mathrm{sec}$ denaturing at $95^{\circ} \mathrm{C}, 5 \mathrm{sec}$ annealing at $60^{\circ} \mathrm{C}$ and a $10 \mathrm{sec}$ extension at $72^{\circ} \mathrm{C}$. These experiments were carried out in triplicate and the mean value was then calculated. The methylation level of the target gene was normalized by $\beta$-actin.

RNA extraction, conversion to $c D N A$ and quantitative reverse transcriptase polymerase chain reaction $(q-R T-P C R)$. The total RNA from 27 paired of non-cancerous mucosa and the matched cancerous lesion (in 2 cases, mRNA was not high quality) was extracted using the Isogen (Nippon Gene, Toyama, Japan) according to the manufacturer's method. A
Table III. Clinicopathological characteristics of patients.

\begin{tabular}{lc}
\hline Factors & $\mathrm{n}(\%)$ \\
\hline Total & $27(100)$ \\
Age & $65.5 \pm 11.4$ \\
Gender & \\
Female & $10(37.0)$ \\
Male & $17(63.0)$ \\
Histological type & \\
tub & $22(81.5)$ \\
por, muc & $5(18.5)$ \\
Dukes & \\
B, C & $15(55.6)$ \\
D & $12(44.4)$ \\
Treatment of S-1/CPT-11 & \\
1st & $15(55.6)$ \\
2nd & $9(33.3)$ \\
3rd & $2(7.4)$ \\
4th & $13(48.1)$ \\
Prior chemotherapy & $15(51.9)$ \\
Tegafur-uracil/LV & \\
I-LV/5-FU & \\
S-1 & \\
Tegafur-uracil & \\
5'-DFUR/CPT-11 & \\
Target disease & \\
Advanced & \\
Recurrence & \\
Non-responder (SD, PD) & \\
\hline
\end{tabular}

500-ng aliquot of RNA was converted into cDNA by reverse transcriptase using a ReverTra Ace ${ }^{\circledR}$ q PCR RT kit (Toyobo, Osaka, Japan) according to the manufacturer's instruction. cDNA was suspended in $50 \mu 1 \mathrm{TE}$ buffer. In order to quanti- 

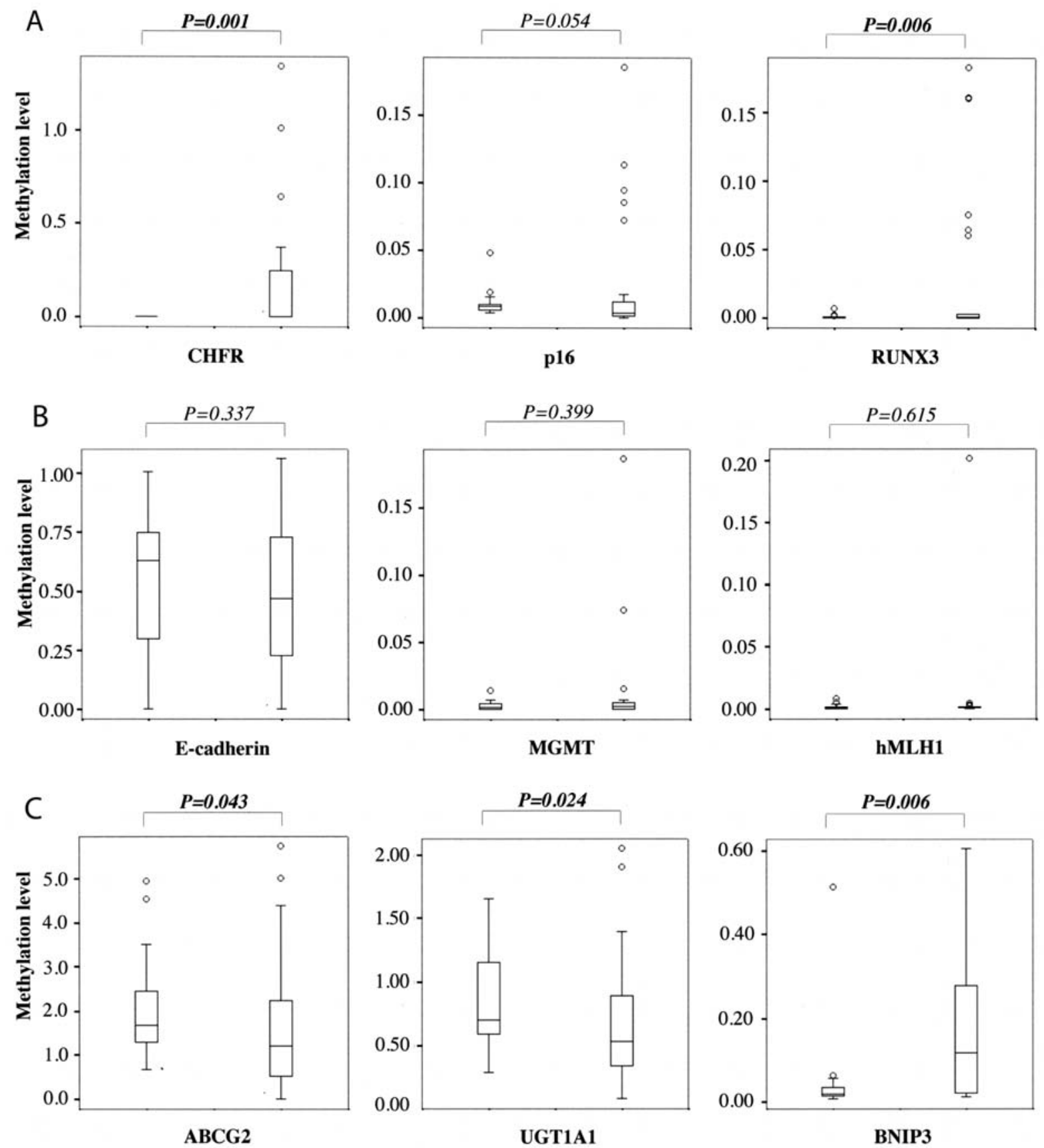

Figure 1. Methylation level of the target gene/ß-actin between non-cancerous (left bar) and primary cancerous specimens (right bar). CHFR, RUNX3 and BNIP3 demonstrated a significantly higher level in cancerous tissue than non-cancerous tissue. ABCG2 and UGT1A1 showed a lower level in cancerous tissue than non-cancerous tissue.

tatively estimate the expression level of mRNA, polymerase chain reaction (PCR) amplification was performed on a Light-Cycler instrument system (Roche) using LightCycler TaqMan Master (Roche) according to the previously report (19). The primer sequences are shown in Table II. After denaturing step at $95^{\circ} \mathrm{C}$ for $10 \mathrm{~min}$, PCR amplification was performed with 45 cycles of $15 \mathrm{sec}$ denaturing at $95^{\circ} \mathrm{C}, 5 \mathrm{sec}$ annealing at $60^{\circ} \mathrm{C}$ and a $10 \mathrm{sec}$ extension at $72^{\circ} \mathrm{C}$. These experiments were carried out in triplicate and the mean value was then calculated. The mRNA expression of the target gene was normalized by GAPDH.

Data analysis and statistics. The statistical analysis was performed using the Mann-Whitney U test or Welch test with the Komlogorv-Sminov test. The cumulative TTP rate was computed according to the Kaplan-Meier method and compared between groups by the log-rank test. Spearman's correlation test was used to assess the correlation between the methylation level and mRNA expression. Statistical analyses were done with StatMate III (ATM Co., Ltd., Tokyo, Japan). A P-value $<0.05$ was considered statistically significant.

\section{Results}

The clinical characteristics of the 27 patients are summarized in Table III. The clinical stages of all patients were grouped as beyond Dukes B at the operation. There were 13 patients in the RG $(48.1 \%)$ and 14 in the NRG $(51.9 \%)$. 

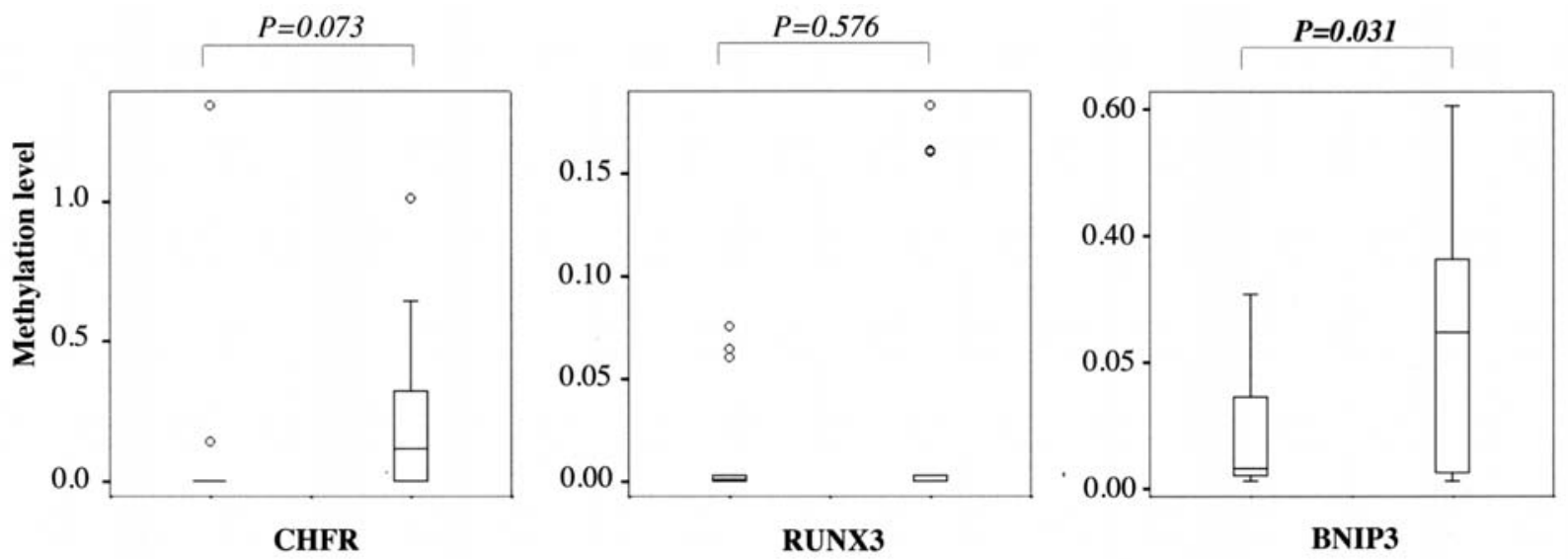

Figure 2. Methylation level between the responder group (RG; left bar) and non-responder group (NRG; right bar). CHFR and RUNX3 did not demonstrate significant differences between the RG and NRG. Only BNIP3 showed a higher level in the NRG than the RG.

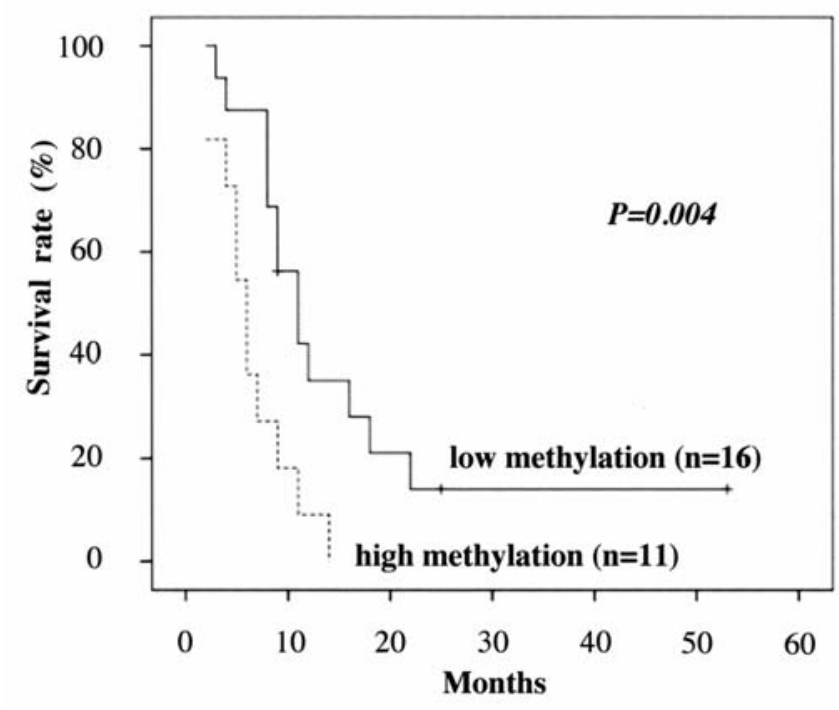

Figure 3. Time to progression curves stratified according to the results of the BNIP3 methylation level. The 27 patients were divided into low and high methylation groups $(n=16, n=11)$ according to the average of the methylation level. A significant difference was observed between the low and high methylation group using the Kaplan-Meier method.

Aberrant gene methylation. The methylation level for 9 genes, CHFR, p16, RUNX3, E-cadherin, MGMT, hMLH1, ABCG2, UGT1A1 and BNIP3 was initially assessed by q-MSP analysis using the 27 paired non-cancerous mucosa and primary cancer specimens (Fig. 1). The methylation level of CHFR, RUNX3 and BNIP3 was significantly higher in the cancer lesions in comparison to the non-cancerous lesion $(\mathrm{P}=0.001, \mathrm{P}=0.006$, $\mathrm{P}=0.006)$. On the contrary, ABCG2 and UGT1A1 showed significantly higher methylation in non-cancerous mucosa than the cancer specimens $(\mathrm{P}=0.043, \mathrm{P}=0.024)$. The methylation level of p16, E-cadherin, MGMT and hMLH1 showed no statistical differences between the non-cancerous and cancer specimens. Based on the results, CHFR, RUNX3, and BNIP3 were determined to carry the cancer specific gene methylation and subjected to further analysis. The methylation level of CHFR, RUNX3 and BNIP3 gene in the cancer specimens was compared between the RG and NRG (Fig. 2).

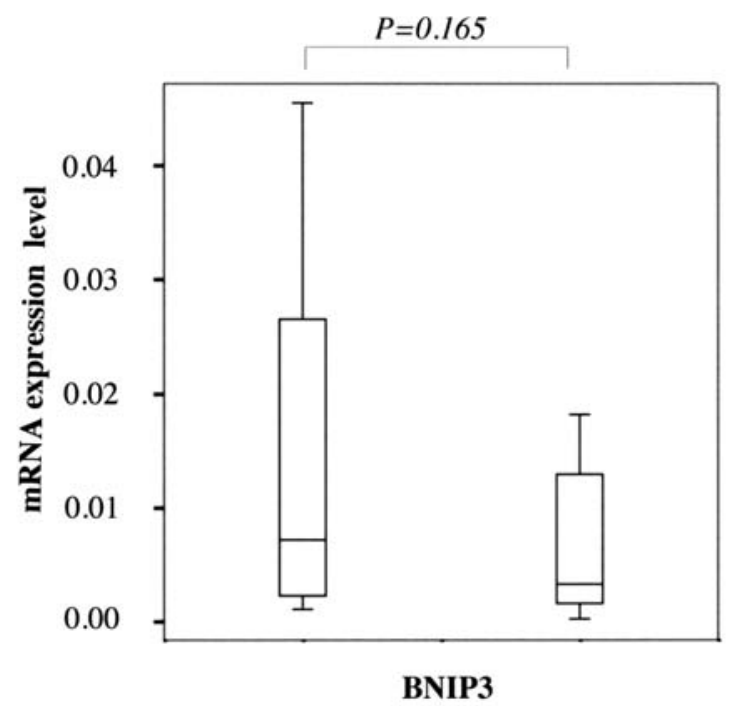

Figure 4. BNIP3 mRNA expression between the responders (left bar) and non-responders (right bar) in the primary cancer lesion. No statistical differences were observed between both lesions. However, the non-responder lesion tended to be lower than responder lesion.

As a result, the methylation level of only BNIP3 was significantly higher in the NRG than in the $\mathrm{RG}(\mathrm{P}=0.031)$.

Correlation between BNIP3 gene methylation and time to progression. The BNIP3 methylation status and TTP were analyzed to assess the efficacy of S-1/CPT-11 therapy. Patients were divided into a high methylation $(n=11)$ and low methylation group $(n=16)$, which was determined according to an average of the methylation level in the cancerous lesion (mean, 0.1651 ; range, $0.0122-0.6037$ ). The result indicated that the low methylation group showed a significantly longer term to TTP, in comparison to the high methylation group $(\mathrm{P}=0.004$; Fig. 3).

BNIP3 expression in cancerous lesion. BNIP3 mRNA expression was quantitatively analyzed in cancer specimens from the 27 CRC patients. The BNIP3 mRNA level tended to be 


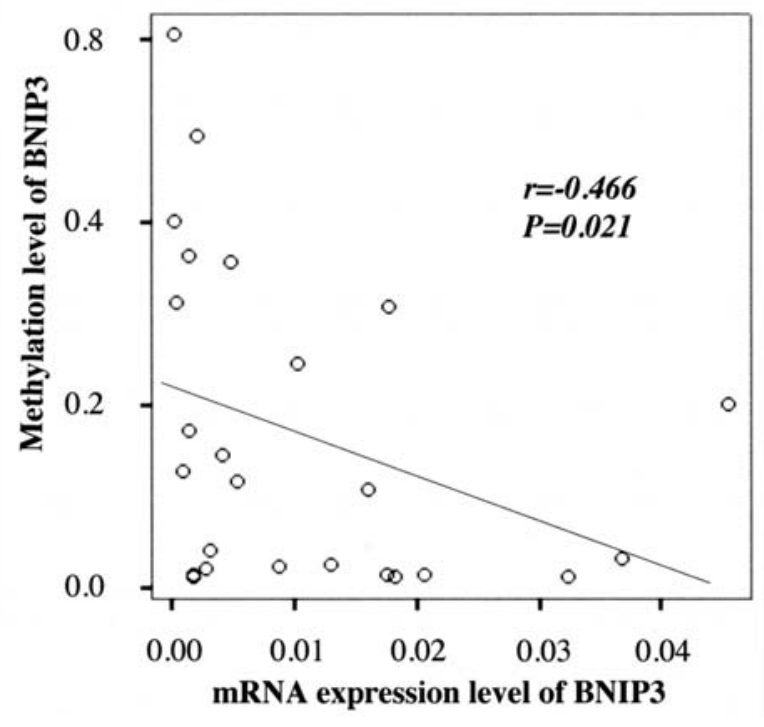

Figure 5. Analysis of the correlation between the levels of BNIP methylation and mRNA expression in primary cancer lesions. Spearman's correlation analysis showed that methylation level was significantly correlated with the mRNA expression.

lower in the NRG than in the RG, although there was no statistically significat difference $(P=0.165$; Fig. 4). In addition, the correlation between methylation and mRNA level was analyzed in the cancer lesions. The methylation level of BNIP3 showed a significant inverse correlation with the mRNA expression ( $\mathrm{r}=-0.466, \mathrm{P}=0.021$; Fig. 5).

\section{Discussion}

Various treatment regimens have been examined in chemotherapy against CRC. A phase I/II study assessed the toxicity and efficacy of S-1/CPT-11 combined therapy for metastatic CRC $(13,25)$. The result showed that a response to the S1/CPT-11 combined therapy was obtained in 19 of 33 (58\%) patients without any significant toxicity, suggesting the efficacy of S-1/CPT-11 against metastatic CRC (13). However, this combination therapy did not provide any therapeutic benefit to the remaining 14 of $33(42 \%)$ patients. If a molecular marker could identify the responders before the treatment, targeting the responders could increase the response rate to $100 \%$. Conversely, another regimen might be considered for the patients identified as non-responder to S-1/ CPT-11 treatment.

$\mathrm{S}-1$, a biochemical modulator of 5-FU, is an oral DPD inhibitory fluoropyrimidine based on the biochemical modulation of 5-FU (26). S-1 was approved in Japan for gastric, colorectal, head and neck, breast, pancreas, biliary tract and lung cancer. On the other hand, CPT-11 is a derivative of camptothecin and a potent DNA topoisomerase I (topo-I) inhibitor that forms stable topo-I DNA-cleavable complexes and inhibits the progression of the replication fork (27). CPT-11 has no cross-resistance with 5-fluorouracil (5-FU) and functions through a novel molecular mechanism (28).

This study analyzed promoter hypermethylation of the cancer related genes in 27 paired non-cancerous mucosa and primary cancers. Nine of the cancer related genes, CHFR, p16, RUNX3, E-cadherin, MGMT, hMLH1, ABCG2,
UGT1A1 and BNIP3, were chosen for methylation analysis, because frequent gene methylation has been reported in solid cancers, including CRC (5-8,10-13,14-17,20-24). A significant correlation between gene methylation or expression and chemosensitivity to 5-FU, S-1 and CPT-11 has been reported for p16 (11), MGMT (29), hMLH1 (12), ABCG2 $(16,30)$, UGT1A1 (14) and BNIP3 (31).

Aberrant gene methylation is frequently observed in the $\mathrm{CpG}$ rich region in the promoter of various cancer related genes in various solid tumors. Gene methylation in cancer cells leads to epigenetic gene silencing and plays an important role in carcinogenesis, cancer progression and drug resistance (5-9). A methylation analysis in cancer tissues has been widely examined by the MSP method. However, the conventional procedure is not able to assess the methylation level in the cancer tissues.

The present study evaluated the methylation levels of 9 cancer related genes by q-MSP method in non-cancerous mucosa as well as cancer lesion. The results demonstrated the methylation level of CHFR, RUNX3 and BNIP3 genes was significantly higher in cancer tissue than in non-cancerous mucosa (Fig. 1). However, p16, E-cadherin MGMT, hMLH1, ABCG2 and UGT1A1 did not show increased methylation in cancer specimens from the 27 patients (Fig. 1). CHFR, RUNX3 and BNIP3, which express CRC specific methylation, were subsequently investigated to determine the correlation with chemosensitivity to S-1/CPT-11 therapy. The BNIP3 methylation level was significantly higher in the non-responders than the responders (Fig. 2). TTP, which represents the efficacy duration of this therapy, was significantly longer in the low methylation group, in comparison to the high methylation. Taken together, these results suggested that BNIP3 gene methylation in the CRC cells decreased the responsiveness to $\mathrm{S}-1 / \mathrm{CPT}-11$ therapy.

Solid tumors often contain a hypoxic region, which is associated with the resistance to chemotherapy and radiation (32). BNIP3 was originally identified as a protein that interacts with adenovirus E1B 19-kDa protein (33). The BNIP3 expression is known to be up-regulated by hypoxia, while also inducing apoptosis in cancer cells (34). However, BNIP3 expression is frequently silenced by DNA methylation and histone deacetylation in CRC $(8,35)$. Abe et al (36) reported that demethylation, using a methyltransferase inhibitor, restores the expression of BNIP3 and induces hypoxiamediated cell death in pancreatic cancer. Erkan et al (31) reported that the down-regulation of BNIP3 resulted in increased resistance to 5-FU and gemcitabine. The authors demonstrated that the loss of BNIP3 expression occurs late in pancreatic cancer and contributes to resistance to chemotherapy, resulting in the worsened prognosis. These results suggested that the aberrant BNIP3 methylation in cancer cells, which suppresses expression, allows the tumors to escape apoptosis in response to hypoxia and chemotherapy. The present study demonstrated the decreased expression of BNIP3 in the NRG in comparison to the RG (Fig. 4). Moreover, the methylation level of BNIP3 was inversely correlated with the mRNA level in the 27 cancer lesions (Fig. 5). These results suggest that the epigenetic gene silencing of BNIP3 by DNA methylation occurs in the CRC tissues and causes the chemoresistance to $\mathrm{S}-1 / \mathrm{CPT}-11$ treatment. 
In conclusion, BNIP3 gene methylation is a possible marker predicting a poor response to S-1/CPT-11 combined therapy in colorectal cancer. Although it is necessary to increase the number of patients analyzed, tailor-made therapy according to the BNIP3 gene methylation might potentially improve the efficacy of S-1/CPT-11 therapy against CRC.

\section{References}

1. Jemal A, Siegel R, Ward E, Hao Y, Xu J, Murray T and Thun MJ: Cancer statistics, 2008. CA Cancer J Clin 58: 71-96, 2008.

2. Ichikawa W, Takahashi T, Suto K, et al: Simple combination of 5-FU pathway genes predict the outcome of metastatic gastric cancer patients treated by S-1. Int J Cancer 119: 1927-1933, 2006.

3. Sato K, Kitajima Y, Miyoshi A, Koga Y and Miyazaki K: Deficient expression of the DPD gene is caused by epigenetic modification in biliary tract cancer cells, and induces high sensitivity to 5-FU treatment. Int J Oncol 29: 429-435, 2006

4. Kai K, Kitajima Y, Hiraki M, et al: Quantitative doublefluorescence immunohistochemistry (qDFIHC), a novel technology to assess protein expression: a pilot study analyzing 5-FU sensitive markers thymidylate synthase, dihydropyrimidine dehydrogenase and orotate phosphoribosyl transferases in gastric cancer tissue specimens. Cancr Lett 258: 45-54, 2007.

5. Toyota M, Ohe-Toyota M, Ahuja N and Issa JP: Distinct genetic profiles in colorectal tumors with or without the $\mathrm{CpG}$ island methylator phenotype. Proc Natl Acad Sci USA 97: 710-715, 2000 .

6. Esteller M, Corn PG, Baylin SB and Herman JG: A gene hypermethylation profile of human cancer. Cancer Res 61: 3225-3229, 2001

7. Esteller M, Toyota M, Sanchez-Cespedes M, et al: Inactivation of the DNA repair gene $\mathrm{O}^{6}$-methylguanine-DNA methyltransferase by promoter hypermethylation is associated with $\mathrm{G}$ to $\mathrm{A}$ mutations in K-ras in colorectal tumorigenesis. Cancer Res 60 : 2368-2371, 2000.

8. Murai M, Toyota M, Suzuki H, et al: Aberrant methylation and silencing of the BNIP3 gene in colorectal and gastric cancer. Clin Cancer Res 11: 1021-1027, 2005.

9. Ohtsuka T, Liu XF, Koga Y, et al: Methylation-induced silencing of ASC and the effect of expressed ASC on p53-mediated chemosensitivity in colorectal cancer. Oncogene 25: 1807-1811, 2006.

10. Koga Y, Kitajima Y, Miyoshi A, Sato K and Miyzaki K: The significance of aberrant CHFR methylation for clinical response to microtubule inhibitors in gastric cancer. J Gastroenterol 41: 133-139, 2006

11. Mitsuno M, Kitajima Y, Ide T, Ohtaka K, Tanaka M, Satoh S and Miyazaki K: Aberrant methylation of p16 predicts candidates for 5-fluorouracil based adjuvant therapy in gastric cancer patients. J Gastroenterol 42: 866-873, 2007.

12. Ide T, Kitajima Y, Ohtaka K, Mitsuno M, Nakafusa Y and Miyazaki K: Expression of the hMLH1 gene is a possible predictor for the clinical response to 5-fluorouracil after a surgical resection in colorectal cancer. Oncol Rep 19: 15711576,2008

13. Nakafusa Y, Tanaka M, Ohtsuka T, et al: Phase I/II study of combination therapy with S-1and CPT-11 for metastatic colorectal cancer. Mol Med Rep 1: 925-930, 2008

14. Gagnon JF, Bernard O, Villeneuve L, Têtu B and Guillemette C: Irinotecan inactivation is modulated by epigenetic silencing of UGT1A1 in colon cancer. Clin Cancer Res 12: 1850-1858, 2006.

15. Kohya N, Koga Y, Kitajima Y and Miyazaki K: Aberrant promoter hypermethylation in biliary tract carcinoma. J Hepatobiliary Pancreat Surg 13: 296-305, 2006.

16. Nakano H, Nakamura Y, Soda $\mathrm{H}$, et al: Methylation status of breast cancer resistance protein detected by methylation-specific polymerase chain reaction analysis is correlated inversely with its ecpression in drug-resistant lung cancer cells. Cancer 112: 1122-1130, 2008.
17. Kitajima Y, Ohtaka K, Mitsuno M, Tanaka M, Sato S, Nakafusa Y and Miyazaki K: Helicobacter pylori infection is an independent risk factor for Runx3 methylation in gastric cancer. Oncol Rep 19: 197-202, 2008.

18. Therasse P, Arbuck SG, Eisenhauer EA, et al: New guidelines to evaluate the response to treatment in solid tumors. European Organization for Research and Treatment of Cancer, National Cancer Institute of the United States, National Cancer Institute of Canada. J Natl Cancer Inst 92: 205-216, 2000.

19. Eads CA, Danenberg KD, Kawakami K, et al: MethyLight: a high-throughput assay to measure DNA methylation. Nucleic Acids Res 28: e32, 2000.

20. Eads CA, Lord RV, Wickramasinghe K, et al: Epigenetic patterns in the progression of esophageal adenocarcinoma. Cancer Res 61: 3410-3418, 2001.

21. Cohen Y, Merhavi-Shoham E, Avraham RB, Frenkel S, Pe'er J and Goldenberg-Cohen N: Hypermethylation of $\mathrm{CpG}$ island loci of multiple tumor suppressor genes in retinoblastoma. Exp Eye Res 86: 201-206, 2008.

22. Herman JG, Graff JR, Myöhänen S, Nelkin BD and Baylin SB: Methylation-specific PCR: a novel PCR assay for methylation status of CpG island. Proc Natl Acad Sci USA 93: 9821-9826, 1996.

23. Esteller M, Gaidano G, Goodman SN, et al: Hypermethylation of the DNA repair gene $\mathrm{O}(6)$-methylguanine DNA methyltransferase and survival of patients with diffuse large B-cell lymphoma. J Natl Cancer Inst 94: 26-32, 2002.

24. Okami J, Simeone DM and Logsdon CD: Silencing of the hypoxia-inducible cell death protein BNIP3 in pancreatic cancer. Cancer Res 64: 5338-5346, 2004.

25. Hiraki M, Yakushiji H, Hashiguchi K and Harada S: Combination chemotherapy with oral TS- 1 and low-dose CPT- 11 by hepatic arterial infusion for multiple hepatic metastases from colon cancer - a case report. Jpn J Cancer Chemother 32: 1055-1058, 2005.

26. Shirasaka T, Shimamato Y, Ohshimo H, Yamaguchi M, Kato T, Yonekura K and Fukushima M: Development of a novel form of an oral 5-fluorouracil derivative (S-1) directed to the potentiation of the tumor selective cytotoxicity of 5 -fluorouracil by two biochemical modulators. Anticancer Drugs 7: 548-557, 1996.

27. Kawato Y, Aonuma M, Hirota Y, Kuga H and Sato K: Intracellular roles of SN-38, a metabolite of the camptothecin derivative CPT-11, in the antitumor effect of CPT-11. Cancer Res 51: 4187-4191, 1991.

28. Rougier P, Bougat R, Douillard JY, et al: Phase II study of irinotecan in the treatment of advanced colorectal cancer in chemotherapy-naive patients and patients pretreated with fluorouracil-based chemotherapy. J Clin Oncol 15: 251-260, 1997.

29. Nagasaka T, Sharp GB, Notohara K, et al: Hypermethylation of $\mathrm{O}^{6}$-methylguanin-DNA methyltransferase promoter may predict nonrecurrence after chemotherapy in colorectal cancer cases. Clin Cancer Res 9: 5306-5312, 2003.

30. Candeil L, Gourdier I, Peyron D, et al: ABCG2 overexpression in colon cancer cells resistant to SN38 and in irinotecan-treated metastases. Int J Cancer 109: 848-854, 2004.

31. Erkan M, Kleff J, Esposito I, et al: Loss of BNIP3 expression in a late event in pancreatic cancer contributing to chemosesistance and worsened prognosis. Oncogene 24: 4421-4432, 2005.

32. Semenza GL: Intratumoral hypooxia, radiation resistance, and HIF-1. Cancer Cell 5: 405-406, 2004.

33. Boyd JM, Malstrom S, Subramanian T, et al: Adenovirus E1B $19 \mathrm{kDa}$ and Bcl-2 protein interact with a common set of cellular proteins. Cell 79: 341-345, 1994

34. Burton TR and Gibson SB: The role of Bcl-2 family member BNIP3 in cell death and disease: NIPping at the heels of cell death. Cell Death Differ 16: 515-523, 2009.

35. Bacon AL, Fox S, Turley H and Harris AL: Selective silencing of the hypoxia-inducible factor 1 target gene BNIP3 by histone deacetylation and methylation in colorectal cancer. Oncogene 26: 132-141, 2007.

36. Abe T, Toyota M, Suzuki H, et al: Upregulation of BNIP3 by 5-aza-2'-deoxycytidine sensitizes pancreatic cancer cells hypoxiamediated cell death. J Gastroenterol 40: 504-510, 2005. 